\title{
Lattice Boltzmann Scheme for Diffusion on Triangular Grids
}

\author{
R.G.M. van der $\operatorname{Sman}^{1,2}$ \\ ${ }^{1}$ Food and Bioprocess Engineering, University of Wageningen, The Netherlands \\ 2 Agrotechnological Research Institute, p.o. box 17, \\ 6700 AA Wageningen, The Netherlands \\ r.g.m.vandersman@ato.dlo.nl
}

\begin{abstract}
In this paper we present a Lattice Boltzmann scheme for diffusion on it unstructured triangular grids. In this formulation of a LB for irregular grids there is no need for interpolation, which is required in other LB schemes on irregular grids. At the end of the propagation step the lattice gas particles arrive exactly at neighbouring lattice sites, as is the case in LB schemes on Bravais lattices. The scheme is constructed using the constraints that the moments of the equilibrium distribution equals that of the Maxwell-Boltzmann distribution. For a special choice of the relaxation parameter $(\omega=1)$ we show that our LB scheme is identical to a cell centered Finite Volume scheme on an unstructured triangular grid.
\end{abstract}

\section{Introduction}

Lattice Boltzmann (LB) has become a powerfull numerical technique for solving complex fluid phenomena, such as multiphase flow and flow in porous media [1]. However, most LB schemes are implemented on uniform structured grids, i.e. Bravais lattices. For many other types of applications this restriction to uniform grids is quite disadvantageous. Several formulations of LB on irregular grids have been developed [2-4], but most of these formulations involve an extra interpolation step, which imposes undesired numerical diffusion.

In a previous paper [5] we have presented a LB scheme for convection diffusion on a rectangular grid with non-uniform lattice spacings, i.e. an irregular, but still structured grid. This scheme is without an extra interpolation step, but adheres the original conceptual framework of the LB schemes on Bravais lattice, where the lattice gas particles always propagate to adjacent lattice sites. In this paper we take a step further in complexity of the grid, and present a LB scheme for diffusion on unstructured triangular grids.

Like in our previous papers $[5,6]$ we show the equivalence between the LB scheme and Finite Volume schemes for the special case of the relaxation parameter $\omega=1$. This equivalence with Finite Volume/Finite Difference has also been noted for LB schemes modelling fluid dynamics on Bravais lattices by Junk [7] and for diffusion by Wolf-Gladrow [8]. In the case of $\omega=1$ the LB scheme is more or less equivalent with the artificial compressibility scheme of Chorin $[7$, 
9]. These insights show that Lattice Boltzmann and Finite Volume can benefit from each other by transfer of concepts. In Finite Volume schemes schemes for irregular unstructured grids are well developed, and can provide good directions how to develop LB schemes for such grids.

For the general case of $\omega \neq 1$ the formulation of the Lattice Boltzmann scheme is not quite identical to that of a scheme for Bravais lattices. We present a proper formulation for a LB scheme describing diffusion on an irregular 1-D grid. It is straightforward to extend this formulation to unstructured triangular grids.

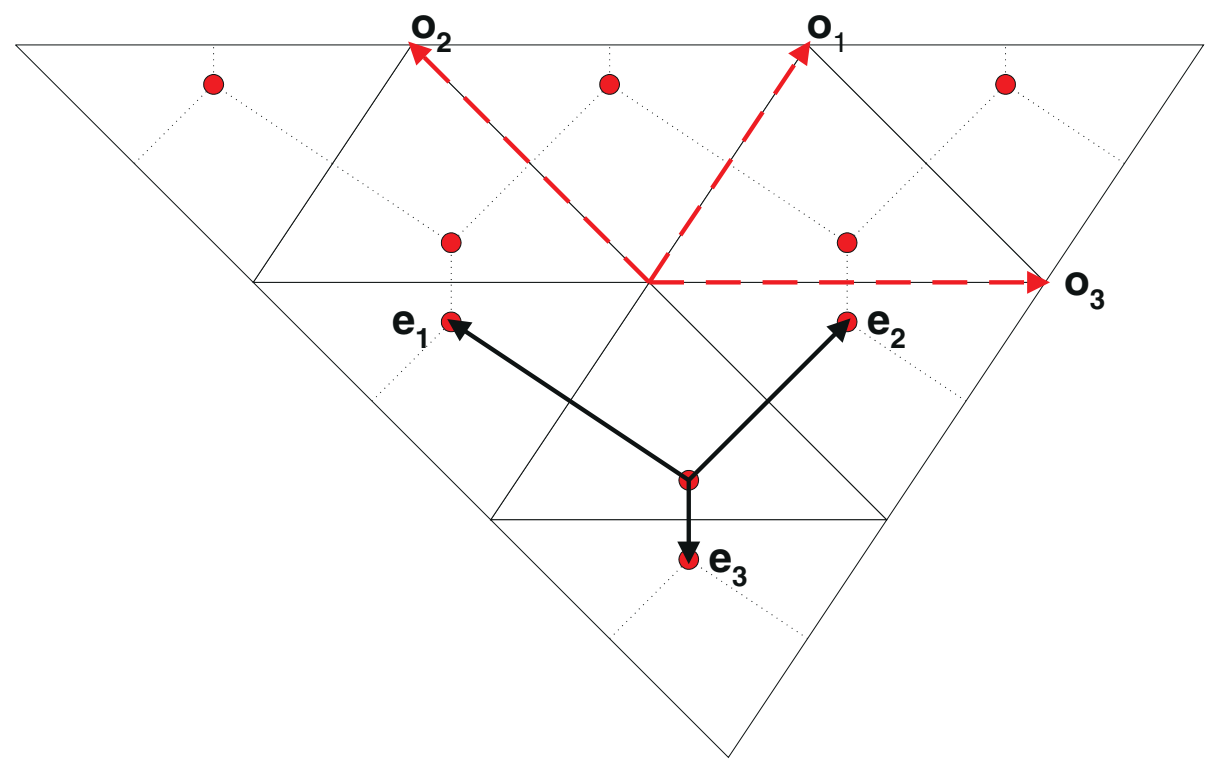

Fig. 1. Grid with triangular lattice cells (control volumes) with lattice sites at the intersection of the orthogonal bisectors $\left\{\mathbf{e}_{i}\right\}$. The vectors $\left\{\mathbf{o}_{i}\right\}$ connect the vertices of the triangles, and form a triangular lattice. The vectors $\left\{\mathbf{e}_{i}\right\}$

form a Voronoi mesh (dashed lines), which is dual to the triangular lattice (solid lines).

\section{Triangular Bravais Lattice}

Before developing the scheme for unstructured triangular grids, it is instructive to develop first the Lattice Boltzmann scheme on a triangular Bravais lattice. For this lattice we assume that the Wigner-Seitz cell is a triangle, with possible unequal sides. The lattice site is located at the intersection of the orthogonal 
bisectors of the triangle. The lattice site is inside the Wigner-Seitz cell if all angles of the triangle are less than $\pi / 2$. This triangular lattice is shown in figure 1.

The vectors connecting the vertices of the Wigner-Seitz cell are defined by:

$$
\begin{aligned}
& \mathbf{o}_{1}=(a, c) \\
& \mathbf{o}_{2}=(-b, c) \\
& \mathbf{o}_{3}=(a+b, 0)
\end{aligned}
$$

The links connecting adjacent lattices sites are denoted as:

$$
\begin{aligned}
& \mathbf{e}_{1}=(-b, d) \\
& \mathbf{e}_{2}=(a, d) \\
& \mathbf{e}_{3}=(0, c-d)
\end{aligned}
$$

with $d=a b / c$, following from the orthogonality of $\mathbf{e}_{i}$ and $\mathbf{o}_{i}$.

By linking the sites one obtains a lattice with Voronoi cells, which is dual to the triangular lattice, defined by the vertices of the Wigner-Seitz cells. Note, this kind of triangular lattice with its dual Voronoi lattice is very common in Finite Elements, where it is named a Delauny triangular grid.

Now we construct the equilibrium distribution following the procedure presented earlier [5], which is based on the constraints for the existence of a global equilibrium distribution. The number of particles at lattice site $\mathbf{x}$ at $\mathbf{x}$ at time t are indicated with $N_{i}(\mathbf{x}, t)$, and the particle density distribution function is $f_{i}(\mathbf{x}, t) \Delta V(x)=N_{i}(\mathbf{x}, t)$. Here $\Delta V$ is the volume of the triangular Wigner-Seitz cell. The velocities of the particles are $\mathbf{c}_{i}=\mathbf{e}_{i} / \Delta t$.

At equilibrium each lattice cell has equal density, and the particle distribution functions are weighted functions of this density:

$$
f_{i}^{e q}=w_{i} \rho
$$

Furthermore, at equilibrium the mass flow rate through each side of the Wigner-Seitz cell is equal to zero. The particles cross the boundary of the lattice cell halfway the links $\left\{\mathbf{e}_{i}\right\}$. Hence, the mass flow rate $J_{i}$ across this boundary is equal to:

$$
J_{i}=\frac{N_{i}^{e q}(\mathbf{x})-N_{i *}^{e q}\left(\mathbf{x}^{*}\right)}{\Delta t}=0
$$

with $\mathbf{x}^{*}=\mathbf{x}+\mathbf{e}_{i}$. With index $i *$ we indicate the velocity of the particle propagating in opposite direction along $\mathbf{e}_{i *}=-\mathbf{e}_{i}$. For a regular grid it follows that $\Delta V(\mathbf{x})=\Delta V\left(\mathbf{x}^{*}\right)$, and consequently

$$
w_{i}=w_{i *}
$$

The lattice gas excert a pressure on all sides of the lattice cell, equal to $p=\rho c_{s}^{2}$, which due to the force exerted by the particles on the side of the Wigner-Seitz cell:

$$
F_{i}=\left[\left\|c_{i}\right\| N_{i}^{e q}(\mathbf{x})+\left\|c_{i *}\right\| N_{i *}^{e q}(\mathbf{x})\right] / \Delta t=\rho c_{s}^{2} \Delta S_{i}
$$


Here $\Delta S_{i}$ is the area (or rather the length in 2-D) of the side of the Wigner-Seitz cell which is normal to $\mathbf{e}_{i}$. Using above definition of the links of the dual lattice, we have $\Delta S_{i}=\left\|\mathbf{o}_{i}\right\|$.

Using Eq.(9) the constraint Eq.(10) is satisfied if:

$$
w_{i}=\frac{c_{s}^{2} \Delta t^{2}\left\|\mathbf{o}_{i}\right\|}{2 \Delta V\left\|\mathbf{e}_{i}\right\|}
$$

With the volume of the triangle being $\Delta V=(a+b) c / 2$, and the definitions of $\mathbf{o}_{i}$ and $\mathbf{e}_{i}$, we obtain the weight factors:

$$
\begin{aligned}
& w_{1}=\frac{c_{s}^{2} \Delta t^{2}}{(a+b) b} \\
& w_{2}=\frac{c_{s}^{2} \Delta t^{2}}{(a+b) a} \\
& w_{3}=\frac{c_{s}^{2} \Delta t^{2}}{c^{2}-a b}
\end{aligned}
$$

From computing the moments of the equilibrium particle density distribution $f_{i}^{e q}=w_{i} \rho$ :

$$
\begin{aligned}
\sum_{i} f_{i}^{e q} & =\rho \\
\sum_{i} e_{i, \alpha} f_{i}^{e q} & =0 \\
\sum_{i} e_{i, \alpha} e_{i, \beta} f_{i}^{e q} & =\rho c_{s}^{2} \delta_{\alpha \beta}
\end{aligned}
$$

we observe that these moments are equal to the moments of the MaxwellBoltzmann distribution for a quiescent fluid. In an earlier paper we have shown that if the Maxwell-Boltzmann constraints hold up to second order, the Lattice Boltzmann scheme will be consistent with diffusion [11].

Because the constraints hold for any admissible triangle (with all angles smaller than $\pi / 2$ ) we expect that also Lattice Boltzmann schemes for irregular grids with triangular lattice cells will be consistent with diffusion with an isotropic diffusivity tensor $D_{\alpha \beta}=D \delta_{\alpha \beta}$. In the following section this hypothesis is analysed further by investigating the equivalence of our Lattice Boltzmann scheme with a Finite Volume scheme formulated for diffusion on an irregular Delauny triangular grid. 


\section{Equivalence with Finite Volume}

In a previous paper [5] we have shown that for convection-diffusion on rectangular Bravais lattices the Lattice Boltzmann scheme is equivalent with a Finite Volume schemes (Lax-Wendroff), if the relaxation parameter $\omega=1$. For this special case the Lattice Boltzmann equation reads:

$$
f_{i *}\left(\mathbf{x}+\mathbf{e}_{i}, t+\Delta t\right)=f_{i}^{e q}(\mathbf{x}, t)
$$

Here we investigate whether this equivalence still holds for diffusion on (irregular) triangular grids. Herbin and Labergerie [10] have presented a Finite Volume scheme for convection diffusion on Delauny triangular grids. We briefly discuss how to construct such a scheme for diffusion.

The Finite Volume scheme solves the diffusion equation for the centre points of triangular control volumes, which is located at the intersection of the orthogonal bisectors of the triangles, similar to the grid shown in figure 1. The Finite Volume scheme solves the discretisation of the diffusion equation integrated over the triangular control volume. Using the Gauss theorem the integrated diffusion equation becomes the mass balance for the triangular control volume:

$$
\partial_{t} M\left(\mathbf{x}_{V}\right)=-\sum_{a} J_{a}\left(\mathbf{x}_{V}\right)
$$

Here $M\left(\mathbf{x}_{V}\right)=\int_{V} \rho(\mathbf{x}) d \mathbf{x}=\bar{\rho}\left(\mathbf{x}_{V}\right) \Delta V\left(\mathbf{x}_{V}\right)$ is the total mass contained in the control volume $V$ at location $\mathbf{x}_{V} . J_{a}\left(\mathbf{x}_{V}\right)$ is the mass flow rate across the side $a$ of the triangular control volume, which is an approximation of the flux through side $a$ :

$$
J_{a}\left(\mathbf{x}_{V}\right)=-\int_{a} D \nabla \rho(\mathbf{x}) \cdot \mathbf{n}_{a} d \sigma_{a}
$$

Here $\mathbf{n}_{a}$ is the outward point normal vector of side $a$.

The mass flow rate is approximated as follows:

$$
J_{a}\left(\mathbf{x}_{V}\right)=-D \frac{\left[\bar{\rho}\left(\mathbf{x}_{V}+\mathbf{e}_{a}\right)-\bar{\rho}\left(\mathbf{x}_{V}\right)\right]}{\left\|\mathbf{e}_{a}\right\|}\left\|o_{a}\right\|
$$

with $\left\|o_{a}\right\|=\int_{a} d \sigma_{a}$ the length of side $a$. Using Euler forward time integration, and the above flux approximation the Finite Volume scheme can be written in Finite Difference form:

$$
\frac{\rho\left(\mathbf{x}_{V}, t+\Delta t\right)-\rho\left(\mathbf{x}_{V}, t\right)}{\Delta t}=\sum_{a} D \frac{\left[\bar{\rho}\left(\mathbf{x}_{V}+\mathbf{e}_{a}\right)-\bar{\rho}\left(\mathbf{x}_{V}\right)\right]}{\left\|\mathbf{e}_{a}\right\| \Delta V\left(\mathbf{x}_{V}\right)}\left\|o_{a}\right\|
$$

To check the equivalence of the above Finite Volume scheme with the Lattice Boltzmann scheme for $\omega=1$, we rewrite our LB equation (14) in Finite Difference form:

$$
\rho\left(\mathbf{x}_{V}, t+\Delta t\right)-\rho\left(\mathbf{x}_{V}, t\right)=\sum_{i>0}\left[f_{i *}^{e q}\left(\mathbf{x}_{V}+\mathbf{e}_{i}, t\right)-f_{i}^{e q}\left(\mathbf{x}_{V}, t\right)\right]
$$


Using the expression of the weight factors Eq.(11) we have:

$$
\frac{\rho\left(\mathbf{x}_{V}, t+\Delta t\right)-\rho\left(\mathbf{x}_{V}, t\right)}{\Delta}=\sum_{i>0} \frac{c_{s}^{2} \Delta t\left\|\mathbf{o}_{i}\right\|}{2 \Delta V\left\|\mathbf{e}_{i}\right\|}\left[\rho\left(\mathbf{x}_{V}+\mathbf{e}_{i}, t\right)-\rho(\mathbf{x}, t)\right]
$$

Observe the similarity of Eq.(20) and Eq.(18). These Finite Difference equations are equivalent if the diffusion coefficient is equal to $D=\frac{1}{2} c_{s}^{2} \Delta t$. This is consistent with the regular expression of the diffusion coefficient in LB schemes (with $\omega=1$ ):

$$
D=c_{s}^{2}\left(\frac{1}{\omega}-\frac{1}{2}\right) \Delta t
$$

Here we have proven that the above defined Lattice Boltzmann scheme is consistent with diffusion (at least for $\omega=1$ ), by its equivalence Finite Volume scheme formulated for diffusion on irregular triangular grids[10]. Evenmore, the flux approximation of the LB scheme and Finite Volume scheme are equivalent, and hence we expect that the expression for the equilibrium distribution also holds for unstructured triangular grids. Because the Finite Volume scheme for unstructured triangular grids is consistent with diffusion upto second order, we expect that the LB scheme with the above formulated equilibrium distribution is also consistent with diffusion even for the general case $\omega \geq 1$. Consequently, even for unstructured grid with the Maxwell-Boltzmann constraints one can construct schemes for isotropic diffusion (upto second order accurate) for irregular triangular grids.

\section{1-D Scheme for Diffusion on Irregular Grid}

In order to obtain a proper formulation of the Lattice Boltzmann equation for irregular grids in the general case of $\omega \neq 1$, we investigate in this section the Lattice Boltzmann scheme for diffusion on an irregular 1-D lattice. We assume a D1Q3 lattice with particle velocities $c_{0}(x)=0, c_{1}(x)$, and $c_{2}(x)$, with in general $c_{1}(x) \neq c_{2}(x)$. The vectors connecting lattice site at $x$ to adjacent lattice sites are denoted as $\mathbf{e}_{1}(x)=+c_{1}(x) \Delta t$, and $\mathbf{e}_{2}(x)=-c_{2}(x) \Delta t$.

Note, that before collision the particles move with velocities $+c_{2}(x)$ and $-c_{1}(x)$, and after propagation they move with $-c_{2}(x)$ and $+c_{1}(x)$. In the text below we will denote the particle velocities in general as $c_{i}(x)$, with the index $i$ referring to the lattice link $e_{i}$ - rather than the velocity direction as is common in Lattice Boltzmann schemes for Bravais lattices.

A next difference with LB schemes for Bravais lattices is that schemes for irregular lattices must be formulated in $N_{i}(x, t)$, the particle number distribution function of particles in a lattice cell moving with velocity $c_{i}$, instead of the particle density distribution $f_{i}(x, t) . N_{i}(x)$ is defined as $N_{i}(x)=f_{i}(x) \Delta V(x)$, with $\Delta V(x)$ is the volume of lattice cell. Note that $\Delta V_{i}(x)$ is proportional to $c_{1}(x)+c_{2}(x)$ :

$$
\Delta V(x)=S\left(c_{1}+c_{2}\right) \Delta t
$$


We derive the Lattice Boltzmann scheme following the procedure proposed in our previous paper [5] and above. From the existence of a global equilibrium with uniform density $\rho(x)=\rho_{0}$ follows:

$$
w_{i}(x) \Delta V(x)=w_{i *}\left(x^{*}\right) \Delta V\left(x^{*}\right)
$$

Remind, that the same constraint for triangular Bravais lattices lead to Eq.(9). For irregular rectangular grids we have found the same relation as Eq.(23). Analysis shows that it holds also for unstructured triangular grids.

From the demand of isotropy of pressure (which is also a second rank tensor like the diffusivity tensor) follows:

$$
w_{i}(x)=\frac{c_{s}^{2}}{c_{i}(x)\left(c_{1}(x)+c_{2}(x)\right)}
$$

Note, that the isotropy constraint for triangular Bravais lattices lead to Eq.(11).

Again with the above weight factors the equilibrium distribution satisfies the Maxwell-Boltzmann constraints Eq.(13).

For the precise formulation of the Lattice Boltzmann equation for irregular lattices we analyse the problem of diffusion in a density field with a constant gradient $\Gamma$ :

$$
\rho(x)=\rho_{0}+\Gamma x
$$

In our previous paper [5] we have taken the ansatz that the non-equilibrium part of the distribution function $f_{i}^{n e q}$ has the same form as for regular lattices:

$$
f_{i}^{n e q}(x)=w_{i}(x) \frac{\mathbf{e}_{i}(x) \Gamma}{\omega}
$$

Note that $f_{i}^{n e q}$ is linear $w_{i}(x) e_{i}(x)$, which is an eigenvector of the collision operator $[11,6]$. Using Eqs. (22-24) we obtain for $i=1,2$ :

$$
N_{i}^{n e q}(x)= \pm \frac{c_{s}^{2} \Gamma \Delta t^{2} S}{2 \omega}
$$

and $N_{0}^{n e q}=0$.

Hence, in the case of a density field with a constant gradient, the nonequilibrium part of the particle number distribution function is independent of the lattice spacing.

The total particle number distribution function associated with the density field with constant gradient, is

$$
N_{i}(x, t)=N_{i}^{e q}(x, t)+N_{i}^{n e q}(x, t)=\Delta V(x) w_{i}(x)\left[\rho_{0}+\Gamma x+\frac{\mathbf{e}_{i}(x) \Gamma}{\omega}\right]
$$

This distribution should be a steady state solution of the Lattice Boltzmann equation. Further analysis of this requirement shows how to construct the algorithm of the Lattice Boltzmann for irregular lattices. 


\section{Algorithm}

Before the collision one computes the projections of the particle number distribution function on the eigenvectors $|1,1,1\rangle$ and $\mid 0,+1,-1>$ of the collision operator, which are associated with the total mass of particles $M$ and the nonequilibrium particle mass flow rate. We define these projections as

$$
\begin{aligned}
M(x, t) & =\sum_{i} N_{i}(x, t) \\
I(x, t) & =N_{1}(x, t)-N_{2}(x, t)
\end{aligned}
$$

The collision process is in effect an relaxation of the non-conserved moment $I$ :

$$
I^{\prime}(x)=(1-\omega) I(x)
$$

Here $\omega$ is the relaxation parameter normally appearing the LBE for Bravais lattices. Note $1-\omega$ is the eigenvalue of the collision operator associated the eigenvector $\mid 0,+1,-1>[11,6]$. For convenience sake we assume the other eigenvalues to be zero. For the propagation the post-collision particle number distribution function is constructed from $M(x)$ and $I^{\prime}(x)$ :

$$
N_{i}^{\prime}(x, t)=w_{i}(x) M(x) \pm \frac{1}{2} I^{\prime}(x, t) \text { for } i=1,2
$$

which is propagated to the adjacent cell

$$
N_{i *}\left(x+\mathbf{e}_{i}(x), t+\Delta t\right)=N_{i}^{\prime}(x, t)
$$

This sequence of collision and propagation should produce a steady state solution:

$$
N_{i}(x, t+\Delta t)=N_{i *}^{\prime}\left(x-\mathbf{e}_{i}(x), t\right)=N_{i}(x, t)
$$

Substitution of the steady state solution Eq.(28) in the above equation gives:

$$
\begin{aligned}
& w_{i}(x) \Delta V(x) \rho(x)-\frac{c_{s}^{2} \Gamma \Delta t S}{2 \omega}= \\
& w_{i *}\left(x^{*}\right) \Delta V\left(x^{*}\right)\left[\rho(x)-\Gamma e_{i}\right]-(1-\omega) \frac{c_{s}^{2} \Gamma \Delta t^{2} S}{2 \omega}
\end{aligned}
$$

Using Eq.(23) we obtain:

$$
w_{i}(x) \Delta V(x) e_{i}=\frac{1}{2} c_{s}^{2} \Delta t^{2} S
$$

and using Eq.(22) we obtain the same expression for $w_{i}$ as stated in Eq.(24). Hence with the above scheme there exists a steady state solution for diffusion in a density field with a constant gradient. 
To determine the diffusion coefficient of our new LB scheme for irregular lattices, we analyse the mass flow rate through the boundaries of the WignerSeitz cell. The mass flow rate should follow Ficks law: $J=-D \Gamma S$. In the above example of a density field with a constant gradient the mass flow rate $J$ is:

$$
\begin{aligned}
& J\left(x+\frac{1}{2} \mathbf{e}_{i}(x), t\right)=\frac{N_{i *}(x *, t)-N_{i}(x, t)}{\Delta t}= \\
& \Delta V(x *) w_{i}(x *)\left[\rho(x)+\Gamma \mathbf{e}_{i}(x)+\frac{\mathbf{e}_{* i}(x *) \Gamma}{\omega}\right]-\Delta V(x) w_{i}(x)\left[\rho(x)+\frac{\mathbf{e}_{i}(x) \Gamma}{\omega}\right]
\end{aligned}
$$

Using Eq.(23) we obtain:

$$
J\left(x+\frac{1}{2} \mathbf{e}_{i}(x), t\right)=-\Gamma S c_{s}^{2}\left[\frac{1}{\omega}-\frac{1}{2}\right] \Delta t
$$

Hence, the diffusion coefficient is accordingly Eq.(21). Note, that Eq.(21) is identical to that of LB schemes for Bravais lattices [11]. From this result we conclude that our LB scheme formulated by Eqs.(30-32) is not much different from LB schemes formulated for Bravais lattices. The only difference between their formulations is that the meaning of the indices of the distribution functions $N_{i}$ is different. In LB schemes for Bravais lattices the indices refer to propagation direction, and in LB schemes for irregular grids the indices refer to particular links between adjacent lattice sites.

\section{Conclusions}

In this paper we have presented the building blocks of a Lattice Boltzmann scheme for diffusion on unstructured triangular grids. This new scheme follows the same concepts as the classical LB schemes for Bravais lattices. During propagation the discrete velocity set of the lattice gas particles move them directly to adjacent lattice sites. Collision is modelling by a relaxation towards an equilibrium distribution. As for all LB schemes on Bravais lattices, the equilibrium distribution can be derived from the Maxwell-Boltzmann constraints [12].

First we have derived the LB scheme and its equilibrium distribution for a triangular Bravais lattice. For the special case of the relaxation parameter $\omega=1$ we have shown the equivalence with a cell-centered Finite Volume scheme for diffusion on triangular grids [10]. The Finite Volume scheme is shown to be consistent with diffusion and accurate upto second order, and even for unstructured grids. Hence, we can conclude that the LB scheme is also consistent with diffusion for unstructured triangular grids.

The formulation of LB schemes for irregular grids is a bit different from LB schemes for Bravais lattices. The scheme should be formulated with particle number distribution functions instead of particle density distribution functions. Furthermore, the indices of the distribution functions refer to particular links, instead of propagation directions. Such a formulation we have presented for an irregular 1-D lattice for the general case of $\omega=1$. The algorithm of the scheme follows the two main steps of LB schemes for Bravais lattices: the particles 
evolve according to collision and subsequent propagation. The collision process is described in the moments representation. Above we have presented only the moments $M$ and $I$ associated with the particle mass density and particle mass flux (which is not conserved). Taking into account higher order moments influence only high order accuracy of the LB scheme [6]. Non-conserved moments (like $I$ ) are relaxed towards zero with a rate equal to the eigenvalue $1-\omega_{n}$ of the associated eigenvector of the equilibrium distribution. From the new moments $M$ and $I^{\prime}$ the post-collision particle distribution function $N_{i}^{\prime}(x, t)$ is constructed, which will be propagated subsequently.

The most important point we like to make is the equivalence of the LB scheme with Finite Volume schemes for the special case of $\omega=1$, which holds even for unstructured triangular grids in the diffusion case. Hence, from the existence of Finite Volume schemes for hydrodynamics on unstructured grids one can conclude that proper LB schemes can be formulated also for hydrodynamics on unstructured grids, which still fit in the conceptual framework of LB schemes for Bravais lattices.

\section{References}

1. S. Chen and G.D. Doolen. Lattice Boltzmann method for fluid flows. Ann. Rev. Fluid Mech. 30: 329-364 (1998).

2. F. Nannelli and S. Succi. The Lattice Boltzmann-equation on irregular lattices. it J. Stat. Phys. 68 (3-4): 401-407 (1992).

3. X. He, and G.D. Doolen, Lattice Boltzmann method on a curvilinear coordinate system: Vortex shedding behind a circular cylinder. Phys. Rev. E, 56, 430-440 (1997).

4. O. Filippova, and D. Haenel. Grid refinement for lattice-BGK models. J. Comput. Phys. 147, 219-228 (1998).

5. Van der Sman, R.G.M., and Ernst M.H., Convection Diffusion Lattice Boltzmann scheme for irregular Lattices, J. Comput. Phys., 160, 1-17 (2000).

6. R.G.M. van der Sman and M.H. Ernst. Gallilean invariant Convection-diffusion Lattice Boltzmann scheme for rectangular lattices. Phys. Rev. E, submitted (2003).

7. M. Junk. A Finite Difference Interpretation of the Lattice Boltzmann method. Numer. Methods Partial Differ. Eq., 17 (4): 383-402 (2001).

8. D. Wolf-Gladrow. A Lattice Boltzmann equation for diffusion. J. Stat. Phys. 79 (5/6): 1023-1032 (1995).

9. A. J. Chorin. A numerical method for solving incompressible viscous flow problems. J. Comput. Phys 2 12- (1967).

10. R. Herbin and O. Labergerie. Finite volume schemes for elliptic and elliptichyperbolic problems on triangular meshes Comp. Meth. Appl. Mech. Eng. 147(12): 85-103 (1997).

11. Van der Sman, R.G.M., and Ernst M.H., Diffusion Lattice Boltzmann scheme on an Orthorhombic Lattice, J. Stat. Phys., 94 (1/2), (1999).

12. G. McNamara and B. Alder, Analysis of the Lattice Boltzmann treatment of hydrodynamics, Physica A, 194, 218-228, (1993). 